\title{
Stress failure plays a major role in the development of high-altitude pulmonary oedema in rats
}

\author{
C. Bai*,f, J. She ${ }^{\star * \#, f}$, A. Goolaerts ${ }^{\star}$, Y. Song ${ }^{+}$, C. Shen ${ }^{\#}$, J. Shen ${ }^{\S}$ and Q. Hong*
}

ABSTRACT: Hypoxia and exertion are considered as the two main factors in the development of high-altitude pulmonary oedema (HAPE), however its pathophysiology remains unclear. Therefore, we established a model in which 32 Sprague-Dawley rats were randomly assigned to normoxic rest, hypoxic rest, normoxic exercise and hypoxic exercise.

An altitude of $4,700 \mathrm{~m}$ was simulated using hypobaric hypoxia, while exercise consisted $48 \mathrm{~h}$ walk with 15-20 min breaks every $4 \mathrm{~h}$. Arterial blood gas, bronchoalveolar lavage (BAL), lung wetto-dry weight (W/D) ratio and histological measurements were conducted on each animal.

In rats exercising in hypoxia, BAL protein and lung W/D ratio were significantly increased but no changes in BAL leukotriene $B_{4}$ and immunoglobulin $M$ were observed. In the same group, lung histology showed typical haemorrhagic lung oedema and disruption of both alveolar epithelium and capillary endothelium while hypoxia or exertion alone only induced slight endothelium and epithelium swelling/disruption.

Our study established a direct link between histological and physiological evidence of HAPElike symptoms and we demonstrated that hypoxia and exertion can synergistically induce HAPElike symptoms in Sprague-Dawley rats without inducing lung inflammation. We therefore propose that alveolar epithelium and capillary endothelium stress failure play a major role in the development of HAPE.

KEYWORDS: Exercise, high-altitude pulmonary oedema, hypobaric hypoxia, rat model, stress failure

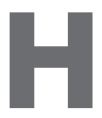
igh-altitude pulmonary oedema (HAPE) is a potentially fatal condition that may affect nonacclimatised individuals who ascend rapidly to altitudes $>3,000 \mathrm{~m}[1,2]$. Approximately $2 \%$ of individuals exposed to high altitude are affected severely enough by HAPE to seek treatment [3]. However, the incidence of sub-clinical HAPE may be as high as $75 \%$ in individuals travelling at an altitude $>4,500 \mathrm{~m}$ [4].

Hypoxia and exertion have been identified as the two main factors in HAPE development [5]. However, the exact mechanisms that trigger the development of HAPE are not completely understood. Although factors such as inflammation and decreased alveolar fluid clearance are thought to be important in HAPE pathophysiology [6-9], pulmonary capillary stress failure, which results from an uneven hypoxic pulmonary vascoconstriction $[10,11]$, has been suggested to be

For editorial comments see page $\mathbf{4 7 0}$.

\section{AFFILIATIONS}

*Dept of Pulmonary Medicine, Zhongshan Hospital, Fudan University,

\# Dept of Pulmonary Medicine, Shanghai Sixth Hospital of Shanghai Jiaotong University, and ${ }^{\S}$ Navy Medical Institute, Shanghai, China

'Dept of Anaesthesia, San Francisco General Hospital, and

${ }^{+}$Dept of Anaesthesia and Perioperative Care, University of California San Francisco, San Francisco, CA, USA.

${ }^{f}$ These authors contributed equally to the present study.

\section{CORRESPONDENCE}

C. Bai

Dept of Pulmonary Medicine, Zhongshan Hospital

Fudan University

180 Feng Lin Road

Shanghai 200032

China

E-mail: bai.chunxue@

zs-hospital.sh.cn

the primary inciting mechanism. Indeed, increased pulmonary capillary transmural pressure [12-14] causes the rupture of the alveolocapillary membrane [15], allowing the flooding of the alveolar space with a protein rich and haemorrhagic oedema fluid. HopKINS et al. [16] found evidence of stress failure in exercising humans under hypoxic conditions, and SWENSON et al. [17] showed the presence of red blood cells as well as increased protein concentration in BAL fluid from humans with HAPE, without significant changes in BAL cytokines. However, whether pulmonary capillary stress failure is the primary mechanism in the development of HAPE is not conclusive since direct evidence of capillary rupture cannot be obtained from humans.

Therefore, an animal model is required to obtain direct histological evidence of capillary rupture in the lung. To date, rabbit, sheep, dogs and ferrets have been used to develop HAPE animal 
models but the results were neither consistent nor reproducible [18-20]. Rats have also been used in a number of studies, but either required endotoxin priming [19], exposure to severe hypoxia (236 Torr, 9,000 m) or the use of specific rat strains such as the Madison strain which has a high susceptibility to the induction of HAPE $[15,21]$. Some studies on pigs showed findings consistent with onset of HAPE when they were exposed to normobaric hypoxia [22], but no convincing histological evidence was provided.

In this study, we hypothesised that the combination of hypoxia and exercise would cause stress failure of the pulmonary capillaries and induce HAPE-like symptoms. In order to test this hypothesis, we established a HAPE model using rats exercising under hypoxic conditions. This model allowed us, for the first time, to directly link histological evidence for capillary breaks to bronchoalveolar lavage (BAL) findings and wet-dry $(\mathrm{W} / \mathrm{D})$ ratios.

\section{MATERIALS AND METHODS Animals}

Adult male Sprague-Dawley rats (Animal Center of Fudan University, Shanghai, China) weighing 252-298 g were used in this study. Rats were exposed to a $12 \mathrm{~h}$ day-night light cycle for 2 days before starting experiments. All animal protocols were approved by the Animal Care Committees of Fudan University. All animal experiments were conducted in accordance with the Guidelines for the Use and Care of Research Animals published by the National Institutes of Health [23].

\section{Experimental design}

Hypoxia setting

A hypobaric hypoxia chamber (Vacuum Chamber, South China Shipbuilding Yard, Shanghai, China) was used to generate specific hypoxic conditions. Briefly, after closing the chamber, air pressure inside the chamber was progressively decreased by a vacuum connected to the chamber. The rate of pressure decrease was set to $20 \mathrm{~m} \cdot \mathrm{s}^{-1}$ until it reached a pressure equivalent to an altitude of $4,700 \mathrm{~m}(\approx 419.97$ Torr $)$.

\section{Rats exercise using treadmill}

Rats were trained to walk on a treadmill (DSPT-202, Duan Co., Ltd, Hangzhou, China) at a speed of $12 \mathrm{~m} \cdot \mathrm{min}^{-1}$ for 2 days before exposure to hypoxia. When the rats were exposed to hypobaric hypoxia, they were forced, using electrical stimulations, to walk on a treadmill at roughly $12 \mathrm{~m} \cdot \mathrm{min}^{-1}$ [24].

\section{Experimental and control groups}

There were eight rats in each group and the groups were as follows: rats exposed to normobaric normoxia at rest (NR); rats exposed to normobaric normoxia with exercise (NE); rats exposed to hypobaric hypoxia with exercise (HE); and rats exposed to hypobaric hypoxia at rest (HR). In the exercise groups (in either hypoxia or normoxia), the rats walked for $48 \mathrm{~h}$ but were allowed to stop for 15-20 min every $4 \mathrm{~h}$, so they could have access to food or water. It is important to note that the rats were, however, continuously exposed to hypoxia during $48 \mathrm{~h}$. All procedures were performed in the main chamber that the investigator could access through an independently controlled antechamber where the pressure was set at 470.48 Torr $(\approx 3,800 \mathrm{~m})$ [21]. When pressure inside the main chamber decreased to $3,800 \mathrm{~m}$, the investigator could enter the main chamber to check the rats. Once the investigator intervention was completed, the chamber was set back at a pressure of 419.97 Torr $(\approx 4,700 \mathrm{~m})$. Thus, the rats were exposed to a simulated altitude of $4,700 \mathrm{~m}$ for most of the time with brief exposures to $3,800 \mathrm{~m}$.

\section{Arterial blood gases}

The following procedure was performed in the main chamber with pressure equivalent to $3,800 \mathrm{~m}$ for investigators safety [21]. After anaesthesia (intraperitoneal injection of $10 \%$ chloral hydrate, $3 \mathrm{mg} \cdot \mathrm{kg}^{-1}$; Sinopharm Chemical Reagent Co., Ltd, Shanghai, China), blood was quickly collected by direct puncture and sampling from the left ventricle using heparinised syringes. All blood samples for arterial blood gas analysis were immediately analysed by GEM Premier 3000 (Instrumentation Laboratory, Bedford, MA, USA). Arterial blood $\mathrm{pH}$, arterial carbon dioxide tension $\left(\mathrm{Pa}_{\mathrm{a}}, \mathrm{CO}_{2}\right)$ and arterial oxygen tension $\left(\mathrm{Pa}_{\mathrm{a}} \mathrm{O}_{2}\right)$ were corrected according to the rectal temperature [22].

\section{Lung $W / D$ ratio}

The rat was placed in a supine position and a cannula was inserted into the trachea after tracheotomy. The lungs were isolated after chest opening, and the right superior lobe was cut to measure wet weight and then dried in an oven for $72 \mathrm{~h}$ in order to get the dry weight. Lung W/D ratio was calculated as described previously $[23,25]$.

\section{Lung histology}

The lungs were inflated with air at pressure of $20 \mathrm{cmH}_{2} \mathrm{O}$ initially then held at $5 \mathrm{cmH}_{2} \mathrm{O}$. After cutting the left atrium, the lungs were perfused with saline via the right ventricle at a pressure of $20-30 \mathrm{cmH}_{2} \mathrm{O}$. When the outflow was clear of blood cells, the lungs were perfused for additional $10 \mathrm{~min}$ and stored in $4 \%$ paraformaldehyde for light microscopy or 3\% glutaraldehyde for electron microscopy at $4^{\circ} \mathrm{C}$ [15]. After 5 days, lungs were embedded and sectioned. Haematoxylin and eosin stained sections were prepared using a standard procedure [23]. The microscopic lung injury was scored based on alveolar oedema, interstitial oedema, haemorrhage, neutrophil and macrophage infiltration. The grades for severity of lung injury in haematoxylin and eosin stained slices were as follows: no injury $=0 ; 25 \%$ of the field injured $=1 ; 50 \%$ of the field injured $=2 ; 75 \%$ of the field injured $=3$; and diffuse injury $=4$ [26]. All histology samples were scored by two independent pathologists following a double blind protocol as done previously [23]. The electron microscopy imaging was performed as described previously [15]. A grading score was set in order to quantify endothelium/epithelium stress failure. This score consisted of the number of breaks observed per surface unit of examined sections.

\section{BAL}

After right lungs were ligated and sectioned, $1 \mathrm{~mL} 0.9 \%$ saline was injected into the left lung through left main bronchus. BAL fluid was withdrawn after 2-3 times gentle and repeated flushing. A total of $3 \mathrm{~mL}$ were injected and BAL fluid was stored in a sterile container, filtered through sterile gauze to remove mucus and cell debris, and then kept at $-80^{\circ} \mathrm{C}$ for later cytokines measurement [22]. The red and white blood cells were counted using Sysmex KX-21 (Sysmex Co., Ltd, Kobe, 
Japan). Total protein and albumin were measured using by an Automatic Analyser (HITACHI-7600, Hitachi Ltd, Tokyo, Japan). BAL immunglobulin (Ig) M was measured by immunoturbidimetry using an Automatic Analyser (HITACHI-7600, Hitachi Ltd) [27]. BAL leukotriene (LT)B $\mathrm{B}_{4}$ was detected by a rat ELISA kit (RapidBio Lab, Calabasas, CA, USA) according to manufacturer's protocol.

\section{Statistical analysis}

Data were presented as mean \pm SEM and analysed using SAS 6.12 software (SAS Institute Inc., Cary, NC, USA). Comparisons between multiple groups were performed using ANOVA with the Bonferroni test [23, 28]. The lung injury scores of histology were analysed by the nonparametric MannWhitney test. $p<0.05$ was considered statistically significant between groups. Pearson test was performed to evaluate data correlation. $\mathrm{r}$ is the correlation coefficient $(-1<\mathrm{r}<1)$. The statistical comparisons were always performed with the control group (NR) unless specified.

\section{RESULTS}

Three out of eight rats exposed to hypobaric hypoxia with exercise died between 27 and $35.5 \mathrm{~h}$. All animals in the other three groups survived.

\section{Arterial blood gases}

We observed a significant decrease of $\mathrm{Pa}_{\mathrm{a}} \mathrm{O}_{2}$ in $\mathrm{HE}$ and $\mathrm{HR}$ rats compared with NR and NE rats (table $1 ; \mathrm{p}<0.01$ ). $\mathrm{Pa}_{1} \mathrm{CO}_{2}$ in $\mathrm{HE}$ and $\mathrm{HR}$ rats was also reduced due to hyperventilation (table 1; $\mathrm{p}<0.01$ and 0.05 , respectively). A significant increase of $\mathrm{pH}$ was only observed in the HR rats (table $1 ; \mathrm{p}<0.05$ ). Based on arterial blood gases, we calculated alveolar-arterial differences for the different groups (table 1). The alveolar-arterial difference was calculated as follows:

$$
\mathrm{PA}, \mathrm{O}_{2}-\mathrm{Pa}, \mathrm{O}_{2}
$$

where

$$
P A, \mathrm{O}_{2}=\left(\mathrm{BP}-\mathrm{PH}_{2} \mathrm{O}\right) \mathrm{FI}, \mathrm{O}_{2}-\mathrm{Pa}_{2} \mathrm{CO}_{2} / \mathrm{R}
$$

$\mathrm{PA}_{1} \mathrm{O}_{2}$ is the alveolar oxygen tension; $\mathrm{BP}$ is the barometric pressure; $\mathrm{PH}_{2} \mathrm{O}$ is water vapour partial pressure at $1 \mathrm{~atm}$ and $37^{\circ} \mathrm{C} ; \mathrm{FI}, \mathrm{O}_{2}$ is inspiratory oxygen fraction; and $\mathrm{R}$ is the respiratory quotient and was assumed at 0.8 in the above equation. These results showed a significant increase in the alveolar-arterial difference of the HE group compared with NR, NE and HR groups, which suggests a decreased efficiency of pulmonary gas exchange.

\section{Lung $W / D$ weight ratio}

Lung W/D ratio is an indicator of overall lung oedema severity [29]. The lung $W / D$ weight ratio in $H E$ and $H R$ rats were significantly higher when compared with control (NR) rats (table $1 ; \mathrm{p}<0.01$ and 0.05 , respectively). The $\mathrm{W} / \mathrm{D}$ ratio of the HE group was significantly higher than the NE group $(p<0.01)$. There was no significant difference in lung W/D weight ratio between NE and NR groups.

\section{BAL}

BAL protein is an indicator of changes in lung alveolarcapillary membrane permeability [30]. The total protein concentration of BAL was increased significantly in HE and
$\mathrm{HR}$ rats compared with $\mathrm{NE}$ and control NR rats (table 1; $\mathrm{p}<0.01$ ). The albumin concentration in BAL from HE and HR rats was also significantly increased compared with $\mathrm{NE}$ and NR rats (table $1 ; \mathrm{p}<0.01$ and 0.05 , respectively). Although the total protein and albumin in $\mathrm{HE}$ and $\mathrm{HR}$ rats were both increased, there was no significant difference between these two groups. Red and white blood cell counts in BAL from HE and HR rats were significantly increased compared with NE and NR rats (table $1 ; \mathrm{p}<0.01$ and 0.05 , respectively). Red blood cell counts in HE rats were, however, significantly higher than in HR rats $(\mathrm{p}<0.05)$. Based on SCHOENE et al. [6] who observed increased levels of BAL IgM and $\mathrm{LTB}_{4}$ in human subjects, we performed BAL IgM and $\mathrm{LTB}_{4}$ measurements. There were no significant differences between the four groups (table 1). BAL $\mathrm{LTB}_{4}$ data suggested that inflammation might not be involved in early stage of our HAPE model. However, BAL protein measurements as well as cell count suggested that hypoxia exposure increased alveolar-capillary permeability, while exercise synergistically amplified the barrier damage caused by hypoxia.

\section{Gross appearance and light microscopy}

Haemorrhagic fluid was noticed in the nose and mouth in three dead rats from the HE group, indicating a severe pulmonary oedema. Three out of five HE survivors and one out of the eight HR rats showed various degrees of lung haemorrhage. Compared with the NR rats (fig. 1a), there were markedly increased alveolar oedema fluid, swollen/thickened pulmonary interstitium and red blood cells in the alveoli of HE nonsurvivors (fig. 1d) and HE survivors rats (fig. 1e). Lung histology in HR rats showed significantly thickened alveolar septum and few red blood cells in alveolar space, but no obvious alveolar oedema (fig. 1c), suggesting that hypoxia per se does not induce HAPE-like symptoms. In NE rats, there was only a slight increase of red blood cells in interstitium, but no significant increase of alveolar and interstitial oedema (fig. 1b). A summary of histological findings is provided in figure 2. Alveolar oedema score in the HE group was significantly increased compared with the other three groups (fig. 2a). Interstitial oedema score in both $\mathrm{HE}$ and $\mathrm{HR}$ groups were significantly increased compared with the NR group (fig. $2 b$; $p<0.01$ ). The haemorrhage score in the HE group was significantly increased compared with NE and NR groups (fig. 2c; $p<0.05$ and 0.01 , respectively). The neutrophil and macrophage infiltration increased in the HE group compared with the control (NR) group (fig. $2 \mathrm{~d}$; $\mathrm{p}<0.05$ ). In summary, morphological analysis showed that combination of hypoxia and exercise induced more severe alveolar-capillary barrier disruption when compared with hypoxia and exercise alone. This severe barrier disruption observed in HE rats was characterised by typical alveolar haemorrhage and oedema.

\section{Electron microscopy}

Electron microscopy was used to show detailed ultra structure changes. An example of normal (from NR group) epithelial and endothelial barrier is shown in figure 3a. In HE rats, there was a complete rupture of the blood-gas barrier (fig. 3d), including disruption of endothelial layer (fig. 3e), swelling of epithelium (fig. 3f) and disruption of epithelium (fig. 3g) compared with control rats (fig. 3a) and NE rats (fig. 3b). The epithelium was heterogeneously affected with either 
TABLE 1 Summary of arterial blood gas analysis, lung wet-dry weight ratio data and bronchoalveolar fluid (BAL) analysis

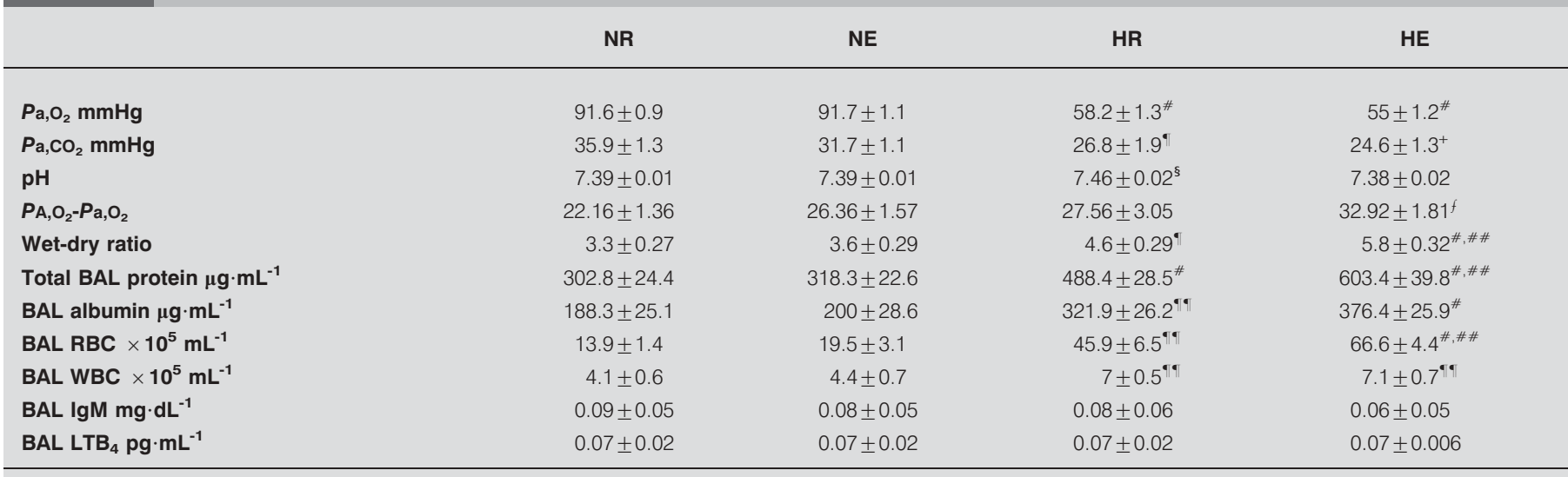

Data are presented as mean \pm SEM. NR: rats exposed to normobaric normoxia at rest; NE: rats exposed to normobaric normoxia with exercise; HR: rats exposed to hypobaric hypoxia at rest; $\mathrm{HE}$ : rats exposed to hypobaric hypoxia with exercise; $\mathrm{Pa}, \mathrm{O}_{2}$ : arterial oxygen tension; $\mathrm{Pa}_{1} \mathrm{CO}_{2}$ : arterial carbon dioxide tension; $\mathrm{PA}_{1} \mathrm{O}_{2}$ : alveolar oxygen tension; RBC: red blood cell; WBC: white blood cell; IgM: immunoglobulin M; LTB 4 : leukotriene $B_{4} .{ }^{\#}: p<0.01$ versus NE and NR groups; ": $p<0.05$ versus NR group; ${ }^{+}: p<0.01$ versus NR group; ${ }^{\text {s: }} p<0.05$ versus HE, NE and NR groups; ${ }^{f}: p<0.05$ versus NR, NE and HR groups; ${ }^{\# \#: ~} p<0.05$ versus HR group; ${ }^{\text {" }}: p<0.05$ versus $\mathrm{NE}$ and NR group. BAL IgM and $\mathrm{LTB}_{4}$ had no significant differences among groups.

evidence of swelling or rupture (fig. $3 \mathrm{f}$ and $\mathrm{g}$ ) and red blood cells in the alveoli suggested the disruption and denudation of alveolar epithelium cells that favoured alveolar flooding. In HR rats, slight endothelium and epithelium swelling as well as a few red blood cells in the alveolar spaces suggested a situation similar to sub-clinical HAPE in humans (fig. 3c). Electron microscopy

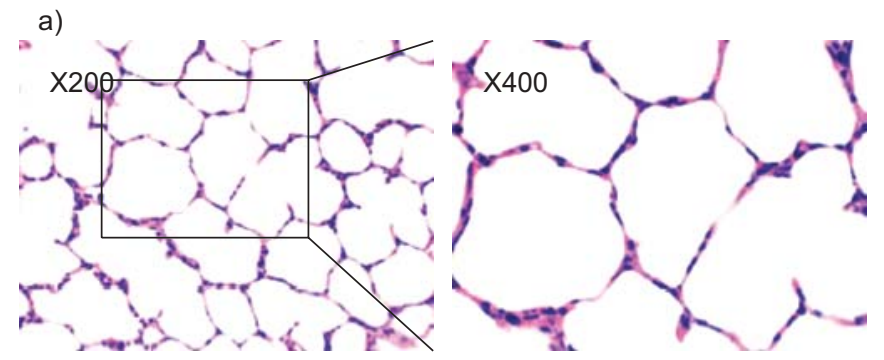

d)
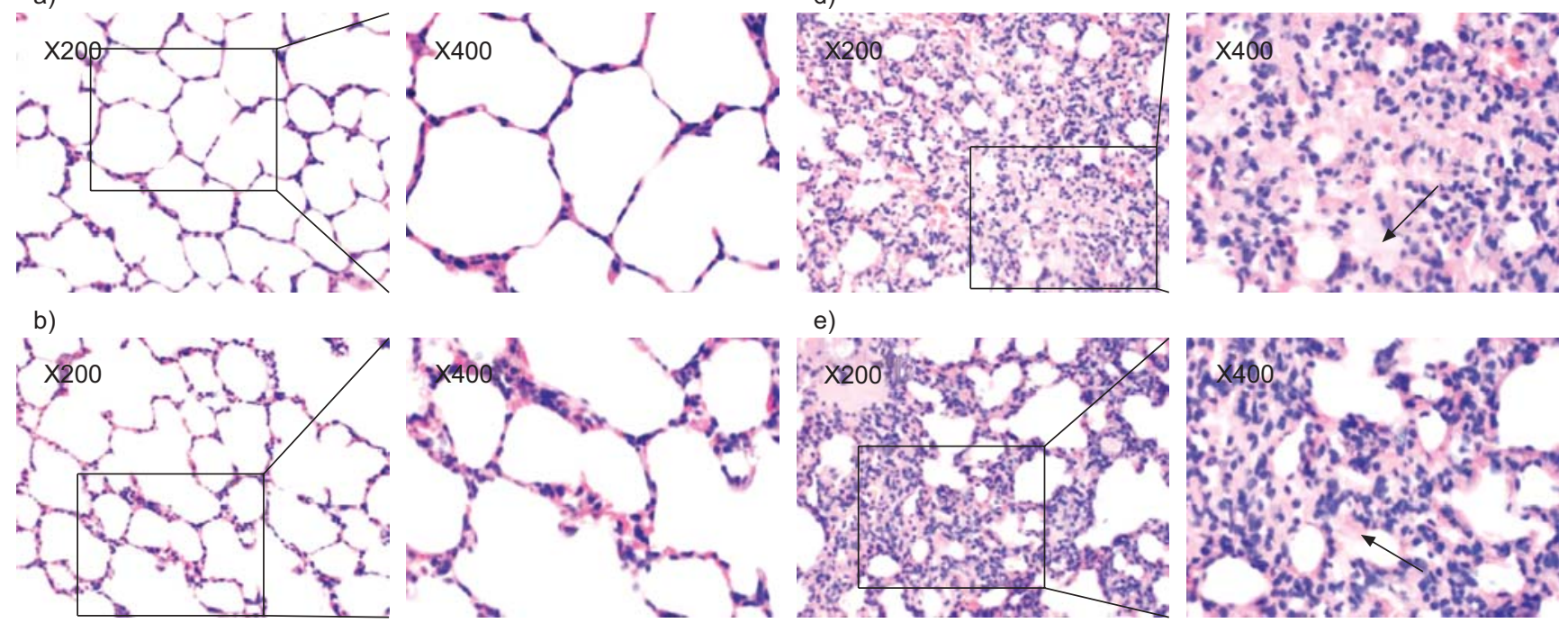

e)
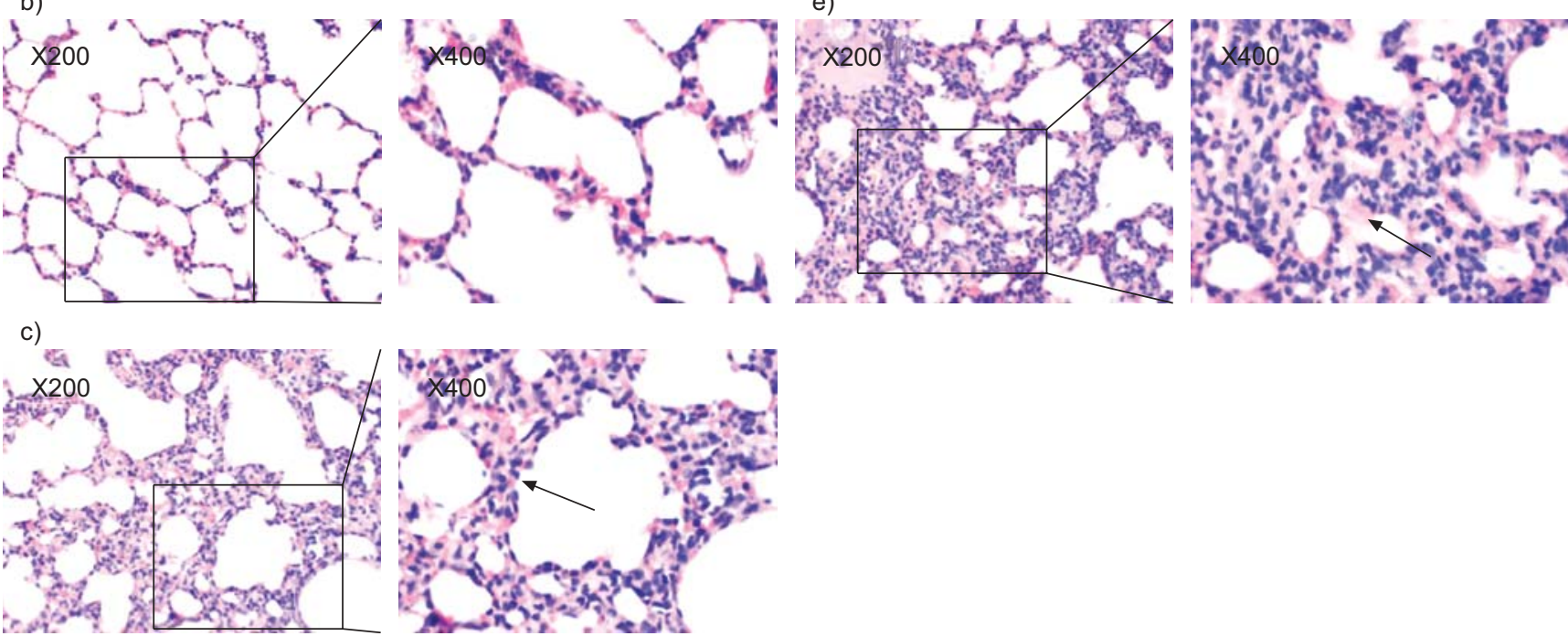

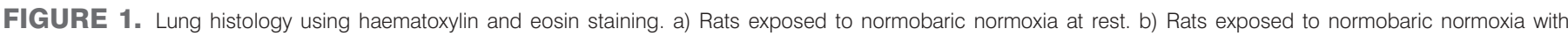

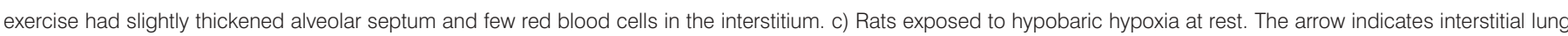

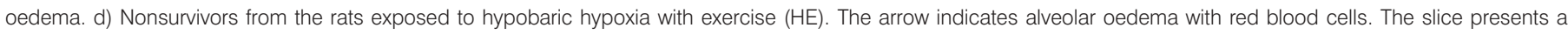

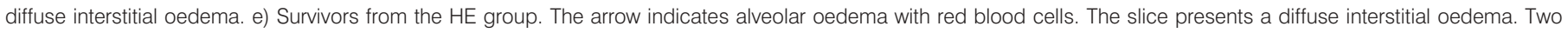
magnifications $(\times 200$ and $\times 400$ ) of the same slice are displayed for each group. 


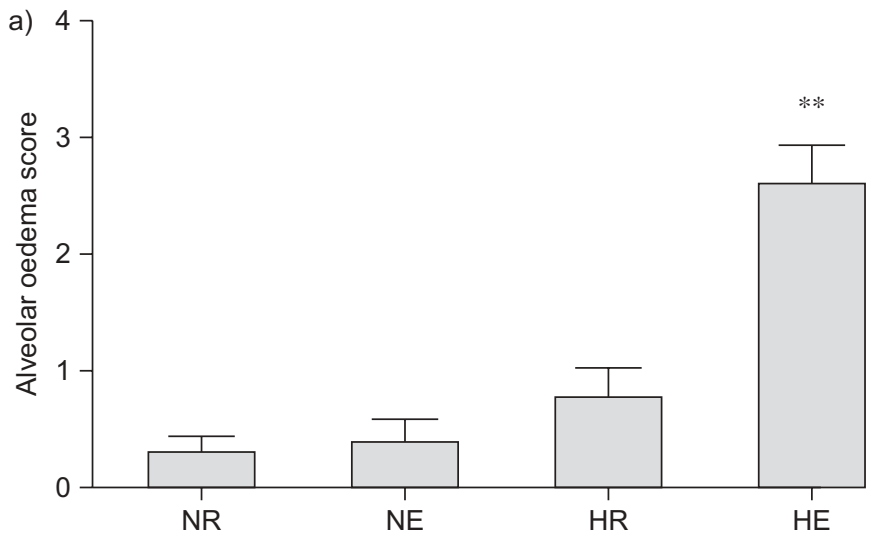

b)
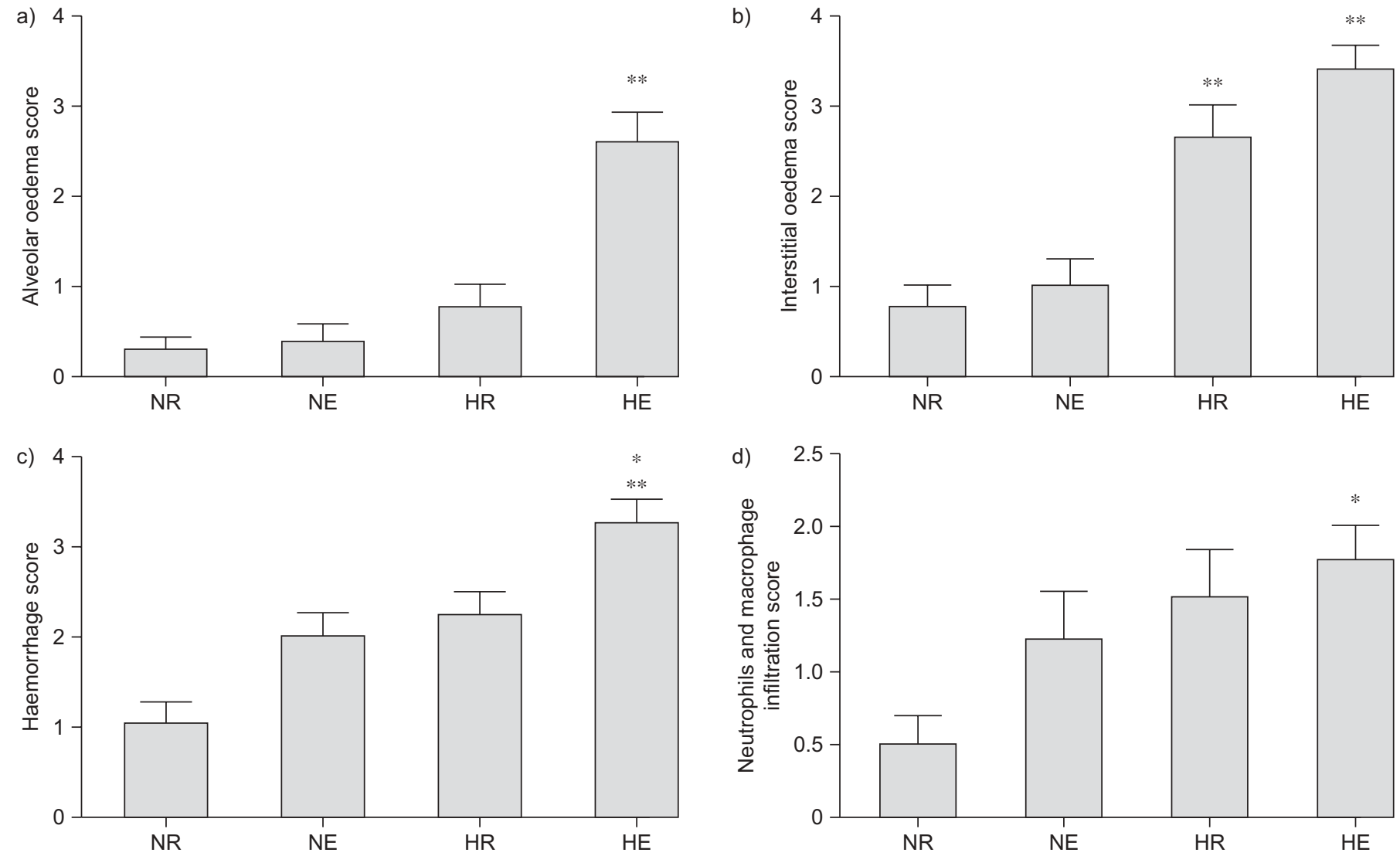

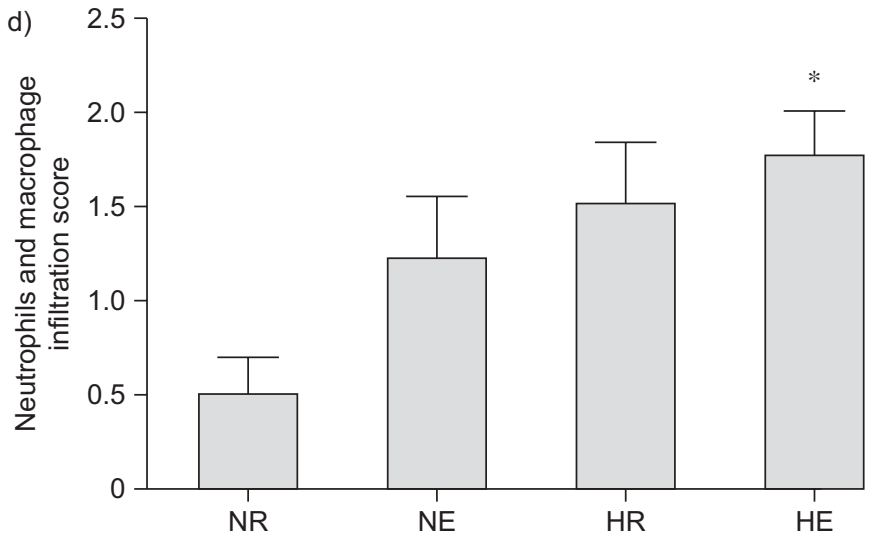

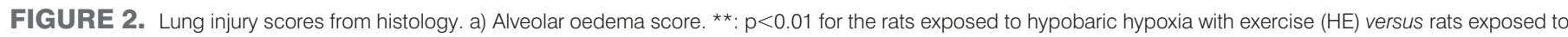

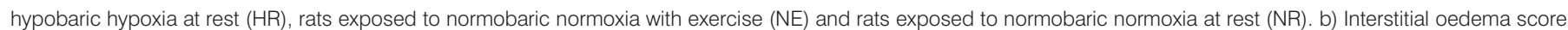

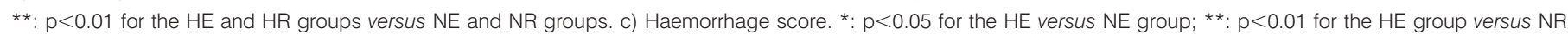
group. d) Neutrophil and macrophage infiltration score. ${ }^{*}: \mathrm{p}<0.05$ for the HE group versus NR group. $n=8$ in each group. Data are presented as mean \pm SEM.

injury score showed that hypoxia or exercise alone only slightly increased endothelial and epithelial cell swelling/disruption, while hypoxia together with exercise significantly induced endothelial and epithelial cell disruption (fig. 3g).

\section{DISCUSSION}

Hypoxia and exertion are considered as the two main factors in the development of HAPE, we hypothesised that HE rats will develop HAPE-like symptoms. Therefore, we established a rat animal model to study the mechanisms of HAPE development due to exercise under hypoxic conditions.

Our results demonstrated that HE rats developed typical HAPE-like symptoms as determined by both physiological and histological analysis such as increased alveolar-arterial difference (table 1), increased $W / D$ ratio (table 1 ), increased BAL total protein/albumin content (table 1), lung endothelial and epithelial cell swelling/disruption and alveolar flooding/ haemorrhage (figs 1, 2 and 3). To our knowledge, this study is the first to show that hypoxia and exercise synergistically induces HAPE-like symptoms in Sprague-Dawley rats. Indeed, although BAL total protein and albumin concentration were significantly increased in rats exposed to either hypoxia or exercise, the increase was higher when rats were simultaneously exposed to both hypoxia and exercise (table 1), suggesting an exacerbation of endothelial/epithelial permeability [31-33]. Also, detailed electron microscopy analysis showed that hypoxia or exercise alone only induces slight alveolar epithelium and capillary endothelium swelling/ disruption, while combined hypoxia and exercise significantly increased endothelium/epithelium swelling and disruption. This destruction of the alveolocapillary membrane by the combination of hypoxia and exertion is underscored by the higher red blood cell count in the BAL from HE rats. In our model, HR rats present intermediate features/symptoms that could correspond to sublinical HAPE in humans. Indeed, the interstitial oedema score, the BAL albumin concentration, the BAL red blood cell content and the $W / D$ ratio are elevated compared with NR and NE groups but these parameters remain lower than that in the HE group and the alveolararterial difference does not indicate an alteration of pulmonary gas exchanges.

Using this model, we also showed for the first time, a direct link between histological evidences for stress failure and other measurements such as W/D ratio, BAL analysis and arterial blood gases. Indeed, capillary breaks positively correlated with oedema score, $\mathrm{W} / \mathrm{D}$ ratio, BAL albumin concentration and the alveolar-arterial difference (table 2). These clear correlations 

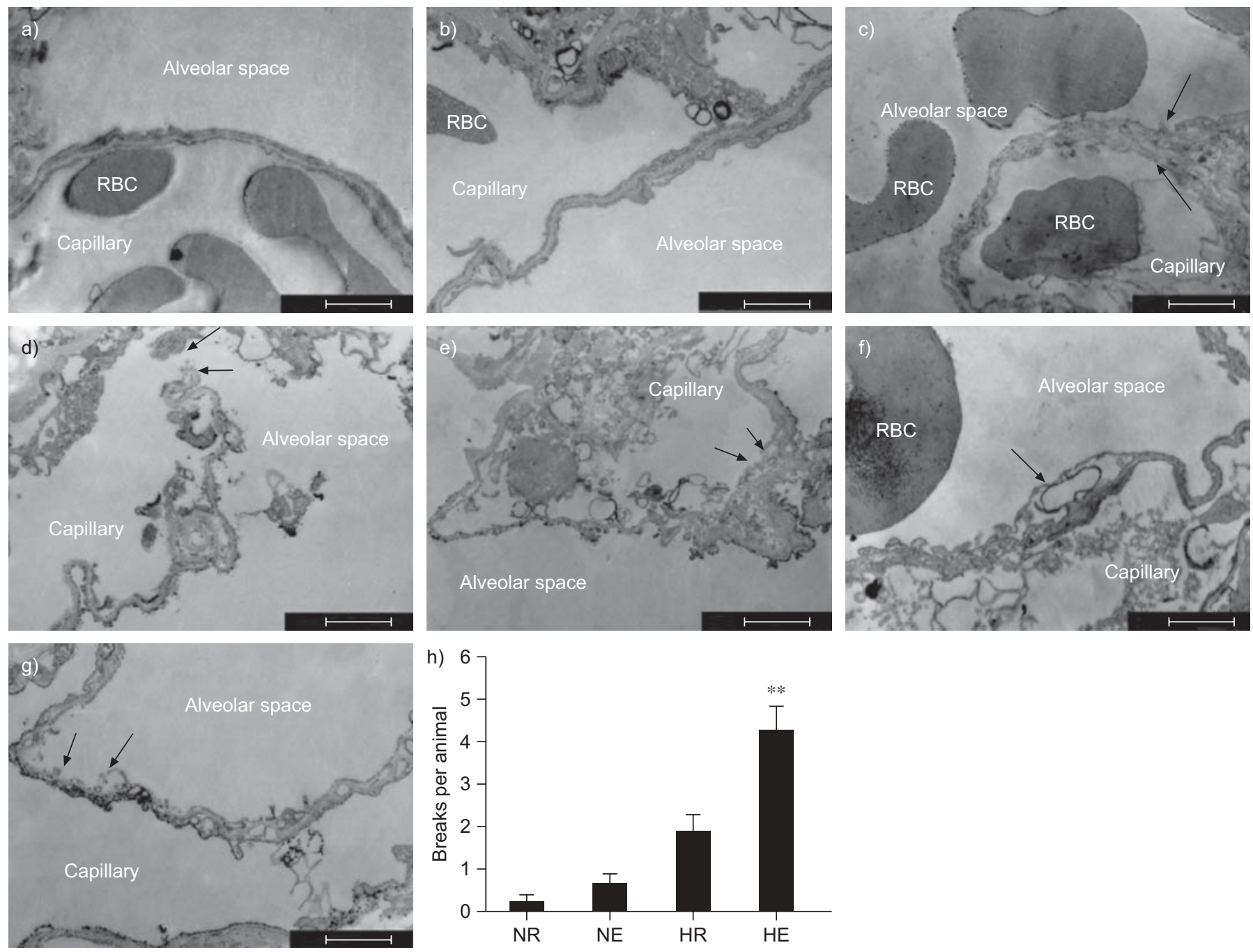

FIGURE 3. Electron microscopy findings. a) Control group (rats exposed to normobaric normoxia at rest (NR)). Continuity of the blood-gas barrier and red blood cells (RBCs) in the capillary. Scale bar $=1 \mu \mathrm{m}$. b) Rats exposed to normobaric normoxia with exercise (NE). Continuity of the blood-gas barrier and RBCs in the capillary. Scale bar $=1 \mu \mathrm{m}$. c) Rats exposed to hypobaric hypoxia at rest (HR). Swelling of endothelial layer and epithelial lining (arrows) and RBCs in the capillary and alveoli. Scale bar $=2 \mu \mathrm{m}$. d) Rats exposed to hypobaric hypoxia with exercise (HE). Complete rupture of the blood-gas barrier including epithelium, endothelium and basement membrane (arrows). Scale bar $=1 \mu \mathrm{m}$. e) HE group. Disruption of endothelial layer (arrows). Scale bar=2 $\mu \mathrm{m}$. f) HE group. Swelling of epithelial lining (arrow) and RBCs in the alveolar space. Scale bar $=2 \mu \mathrm{m} . \mathrm{g}) \mathrm{HE}$ group. Disruption of epithelial lining (arrows). Scale bar=1 $\mu \mathrm{m}$. h) Summary of electron microscopy findings. The results are averaged numbers ( \pm SEM) of alveolocapillary disruption observed per surface unit of slice. Three slices were analysed for each rat. ${ }^{* *}: p<0.01$ for the HE versus HR, NE and NR groups. Lanthanum nitrate was used as a tracer to highlight the alveolocapillary wall.

strongly suggest that stress failure plays a major role in the development of HAPE-like symptoms in our model.

Another factor that has been proposed to play a role in the pathogenesis of HAPE is inflammation [6]. Thus, we measured IgM and $\mathrm{LTB}_{4}$ in BAL. However, no significant difference appears among the four groups for both $\mathrm{LTB}_{4}$ and IgM. The observation of distal lung lesions with no evidence of inflammation suggests that inflammation plays a minor role in the development of HAPE-like symptoms in our animal model [17]. Moreover, although the neutrophile and macrophage infiltration score was higher in the HE group, it was also elevated in the other experimental groups (HR and NE rats) and did not significantly correlate with either the oedema score, the capillary breaks or the $\mathrm{W} / \mathrm{D}$ ratio (table 2).
Decreased alveolar fluid clearance (AFC) has also been proposed to contribute to the development of HAPE [7-9]. Indeed, in case of alveolar flooding, the alveolar fluid clearance constitutes the first line of defence by allowing alveolar fluid removal, a mechanism known to depend on active vectorial ion transport through the epithelium. Due to hypoxia mediated inhibition of function/expression of the alveolar epithelial sodium channel [7, 9], AFC will likely be affected in our model. However, regarding the lung lesions observed in the HE group, we do not think that decreased AFC triggers the formation of HAPE in our model. AFC is currently under investigation in our animal model.

Our model does, however, have some limitations. Indeed, we could not measure pulmonary artery pressure due to 
TABLE 2 Correlation table among bronchoalveolar lavage (BAL) measurements and histological parameters

\begin{tabular}{|c|c|c|c|c|}
\hline & $P A-\mathrm{a}, \mathrm{O}_{2}$ & $\mathrm{BAL} \mathrm{LTB}{ }_{4} \mathrm{pg} \cdot \mathrm{mL}^{-1}$ & Breaks SEM & Neutrophil infiltration score \\
\hline Oedema score & $0.78^{*}$ & 0.21 (NS) & $0.9^{* *}$ & 0.34 (NS) \\
\hline Wet/dry ratio & $0.81^{*}$ & 0.24 (NS) & $0.9^{\star \star}$ & 0.35 (NS) \\
\hline BAL albumin $\mu \mathrm{g} \cdot \mathrm{mL}^{-1}$ & $0.80^{*}$ & 0.29 (NS) & $0.89 * *$ & \\
\hline BAL RBC $\times 10^{5} \mathrm{~mL}^{-1}$ & $0.78^{*}$ & 0.16 (NS) & $0.92^{* \star}$ & \\
\hline
\end{tabular}

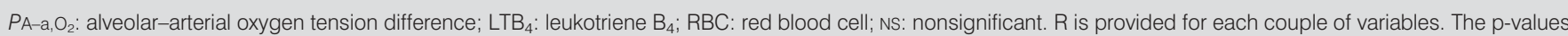
have been calculated using the Pearson test and are provided for each couple of variables. Breaks are the average number of breaks observed per animal in electron microscopy. ${ }^{* *}: p<0.01$.

measurement complexity in our experimental setting and the exercise intensity may not transpose to exertion levels observed in humans.

We therefore propose that exacerbation of hypoxic pulmonary vasoconstriction by exertion would induce stress failure of the distal lung structure, promoting alveolar flooding with a protein rich and haemorrhagic oedema.

In conclusion, our model of exercising rats exposed to hypobaric hypoxia is able to induce HAPE-like symptoms without endotoxin priming, severe hypoxia or the use of a special strain. Our HAPE model is not associated with a lung inflammation. The correlation between elevated albumin concentration in the BAL as well as higher $\mathrm{W} / \mathrm{D}$ ratio and increased endothelium/epithelium swelling/disruption suggested that alveolocapillary barrier stress failure plays a major role in the development of HAPE.

\section{SUPPORT STATEMENT}

The present study was supported by Shanghai Leading Academic Discipline Project (Project Number: B115), the National Natural Science Foundation of China (30871131) and the National 211 Project (211XK20; all China).

\section{STATEMENT OF INTEREST}

None declared.

\section{ACKNOWLEDGEMENTS}

The present authors would like to thank M. Liu (Shanghai Jiaotong University, Shanghai, China) for document retrieval support, Z. Yu (Shanghai Medical School, Fudan University, Shanghai) for electron microscopy technical support, Z. Xu (Shanghai Medical School, Fudan University) for histology support, and H. Zhang and W. Zhu (Navy Medicial Institute, Shanghai) for using hypobaric hypoxia chamber. We also want to thank M. Howard (University of California, San Francisco, CA, USA) for her support in scientific writing.

\section{REFERENCES}

1 West JB. The physiologic basis of high-altitude diseases. Ann Intern Med 2004; 141: 789-800.

2 Mortimer H, Patel S, Peacock AJ. The genetic basis of high-altitude pulmonary oedema. Pharmacol Ther 2004; 101: 183-192.

3 Hackett PH, Rennie D. The incidence, importance, and prophylaxis of acute mountain sickness. Lancet 1976; 2: 1149-1155.
4 Cremona G, Asnaghi R, Baderna P, et al. Pulmonary extravascular fluid accumulation in recreational climber: a prospective study. Lancet 2002; 359: 303-309.

5 Basnyat B, Murdoch DR. High altitude illness. Lancet 2003; 361: 1967-1974.

6 Schoene RB, Swenson ER, Pizzo CJ, et al. The lung at high altitude: bronchoalveolar lavage in acute mountain sickness and pulmonary edema. J Appl Physiol 1988; 64: 2605-2613.

7 Sartori C, Allemann Y, Duplain H, et al. Salmeterol for the prevention of high altitude pulmonary edema. N Engl J Med 2002; 346: 1631-1636.

8 Scherrer U, Sartori C, Lepori M, et al. High-altitude pulmonary edema: from exaggerated pulmonary hypertension to a defect in transepithelial sodium transport. Adv Exp Med Biol 1999; 474: 93-107.

9 Zhou G, Dada LA, Sznajder JL. Regulation of alveolar epithelial function by hypoxia. Eur Respir J 2008; 31: 1107-1113.

10 Grunig E, Mereles D, Hildebrandt W, et al. Stress Doppler echocardiography for identification of susceptibility to high altitude pulmonary edema. I Am Coll Cardiol 2000; 35: 980-987.

11 Dehnert C, Grunig E, Mereles D, et al. Identification of individuals susceptible to high-altitude pulmonary oedema at low altitude. Eur Respir J 2005; 25: 545-551.

12 Maggiorini M, Melot C, Pierre S, et al. High-altitude pulmonary edema is initially caused by an increase in capillary pressure. Circulation 2001; 103: 2078-2083.

13 West JB, Mathieu-Costello O, Prediletto R. Stress failure in pulmonary capillaries. J Appl Physiol 1991; 70: 1731-1742.

14 Tsukimoto K, Mathieu-Costello $\mathrm{O}$, Prediletto $\mathrm{R}$, et al. Ultrastructural appearances of pulmonary capillaries at high transmural pressure. J Appl Physiol 1991; 71: 573-582.

15 West JB, Colice GL, Lee Y-J, et al. Pathogenesis of high-altitude pulmonary oedema: direct evidence of stress failure of pulmonary capillaries. Eur Respir J 1995; 8: 523-529.

16 Hopkins S, Garg J, Bolar DS, et al. Pulmonary blood flow heterogeneity during hypoxia and high-altitude pulmonary edema. Am J Respir Crit Care Med 2005; 171: 83-87.

17 Swenson ER, Maggiorini M, Mongovin S, et al. Pathogenesis of high-altitude pulmonary edema: inflammation is not an etiologic factor. JAMA 2002; 287: 2228-2235.

18 Schoene RB, Goldberg S. The quest for an animal model of high altitude pulmonary edema. Int J Sports Med, 1992: S59-S61.

19 Ono S, Westcott JY, Chang SW, et al. Endotoxin priming followed by high altitude causes pulmonary edema in rats. J Appl Physiol 1993; 74: 1534-1542.

20 Irwin DC, Rhodes J, Baker DC, et al. Atrial natriuretic peptide blockade exacerbates high altitude pulmonary edema in endotoxin primer rat. High Altitude Med Biol 2001; 2: 349-360. 
21 Engebretsen BJ, Irwin D, Valdez ME, et al. Acute hypobaric hypoxia $(5486 \mathrm{~m})$ induces greater pulmonary HIF-1 activation in Hilltop compared to Madison rats. High Altitude Med Biol 2007; 8: 312-321.

22 Kleinsasser A, Levin DL, Loeckinger A, et al. A pig model of high altitude pulmonary edema. High Altitude Med Biol 2003; 4 465-474.

23 Sun JY, Guo WG, Ben Y, et al. Preventive effects of curcumon and dexamethasone on lung transplantation-associated lung injury in rats. Crit Care Med 2008; 36: 1205-1213.

24 Pottle D, Gosselin LE. Impact of mechanical load on functional recovery after muscle reloading. Med Sci Sports Exerc 2000; 32: 2012-2017.

25 Su X, Song YL, Jiang JJ, et al. The role of aquaporin-1 (AQP1) expression in a murine model of lipopolysaccharide-induced acute lung injury. Respir Physiol Neurobiol 2004; 142: 1-11.

26 Su X, Wang L, Song YL, et al. Inhibition of inflammatory responses by ambroxol, a mucolytic agent, in a murine model of acute lung injury induced by lipopolysaccharide. Intensive Care Med 2004; 30 133-140.
27 Riches PG, Sheldon J, Smith AM, et al. Overestimation of monoclonal immunoglobulin by immunochemical methods. Ann Clin Biochem 1991; 28: 253-259.

28 She J, Sun Q, Fan L, et al. Association of HLA genes with diffuse panbronchiolitis in Chinese Patients. Respir Physiol Neurobiol 2007; 157: 266-273.

29 Michel RP, Hakim TS, Smith TJ, et al. Quantitative morphology of permeability lung edema in dogs induced by alphanaphthylthiourea. Lab Invest 1983; 49: 412-419.

30 Briot R, Bayat S, Anglade D, et al. Monitoring the capillary-alveolar leakage in an ARDS model using broncho-alveolar lavage. Microcirculation 2008; 15: 237-249.

31 Ganter MT, Rou J, Miyazawa B, et al. Interleukin-1 $\beta$ causes acute lung injury via $\alpha v \beta 5$ and $\alpha v \beta 6$ integrin-dependent mechanisms. Circ Res 2008; 102: 804-812.

32 Pittet JF, Griffiths MJ, Geiser T, et al. TGF- $\beta$ is a critical mediator of acute lung injury. J Clin Invest 2001; 107: 1537-1544.

33 Dehnert C, Berger MM, Mairbaurl $\mathrm{H}$, et al. High altitude pulmonary edema: a pressure-induced leak. Respir Physiol Neurobiol 2007; 158: 266-273. 